\title{
EFECTO DE UN PROGRAMA EDUCATIVO EN EL CONOCIMIENTO Y LA INCERTIDUMBRE DE LOS FAMILIARES DE PACIENTES EN UNIDAD DE CUIDADOS INTENSIVOS MATERNA
}

\author{
Mariela Zamora Córdova ${ }^{1, a}$, Orlando Meza Curo ${ }^{1, a}$, Elizabeth Juárez Ramos ${ }^{1, a}$, Maritza Salazar Cárdenas $1, a$
}

\begin{abstract}
RESUMEN
Objetivos. Determinar el efecto de un programa educativo, en el nivel de conocimientos y el nivel de incertidumbre de familiares de pacientes críticas Materiales y métodos. Estudio cuasi experimental, prospectivo, participaron 60 familiares directos de las pacientes ingresadas a la UCI Materna. Para la recolección de datos se aplicó como técnica la encuesta, y como instrumento para medir el efecto del programa educativo un cuestionario que evalúa el nivel de conocimientos y el nivel de incertidumbre del familiar de la paciente crítica, cuya validación presento un coeficiente de confiabilidad de 78.8\% medido mediante el Alfa de Cronbach, lo cual garantizó la fiabilidad de la escala usada. Resultados. El nivel de conocimientos medido en familiares de pacientes críticas antes de la aplicación del programa educativo fue del $38.3 \%$ y el nivel de incertidumbre fue alto, $66.7 \%$ de los familiares. El nivel de conocimientos después de la aplicación del programa educativo fue con mayor frecuencia alto $73.3 \%$, y nivel de incertidumbre bajo en el $66.7 \%$ de los familiares. Conclusiones. Se hace necesario la aplicación de un programa educativo como una estrategia efectiva dirigida a los familiares de pacientes ingresados en cuidados intensivos para mejorar el nivel de conocimiento e incertidumbre al encontrarse en un ambiente altamente tecnificado y con procedimientos que aparentan ser cruentos y amenazantes para ellos.
\end{abstract}

Palabras claves: Conocimientos; Incertidumbre; Programa educativo; Paciente crítico (Fuente: DeCS BIREME).

\section{EFFECT OF AN EDUCATIONAL PROGRAM ON KNOWLEDGE AND UNCERTAINTY OF PATIENT FAMILY MEMBERS IN A UNIT OF MATERIAL INTENSIVE CARE}

\begin{abstract}
Objectives. To determinate the effect of an educational program on the level of knowledge and uncertainty in family members of critical patients. Materials and methods. Quantitative research approach, quasi-experiment, perspective, and crosssectional design, was taken to the entire community, consisting of 60 direct relatives of the patients admitted to the $\mathrm{UCl}$ Maternal (Intensive Care Unit). In order to collect the data from the patients, a survey needed to be given to the relatives as a technique, and as a tool, a questionnaire that evaluated the level of knowledge and the level of uncertainty of the family members of the critical patient. Results. the level of awareness in relatives of critical patients before the application of the educational program was at a $38.3 \%$ medium level, and the level of uncertainty was higher by $66.7 \%$ familiar. Also, The level of knowledge after the application of the educational program was $73.3 \%$ above, and the level of uncertainty was below by $66.7 \%$. Conclusions. it is necessary to apply for an educative program as an effective strategy aimed at the relatives of patients admitted to intensive care, in order to improve knowledge and uncertainty, because they are in a highly technical environment and with procedures that appear to be threatening to them.
\end{abstract}

Keywords: knowledge; Uncertainty; Educational program; Critical patient (Source: MeSH NLM).

\section{INTRODUCCIÓN}

La familia es importante en cada ser humano y ésta ve resquebrajada en su tranquilidad a causa de la enfermedad de uno de ellos. La salud familiar es considerada de interés en la salud pública, tanto en el Perú como a nivel internacional.

La creciente demanda de pacientes hospitalizados en unidades de cuidados intensivos, trae consigo familiares que atraviesan estados emocionales amenazados por la incertidumbre y falta de información ${ }^{1}$. Teniendo en cuenta que la familia surge en la vida del hombre como una necesidad de supervivencia humana, con la ruptura de ésta, su estabilidad se ve amenazada ${ }^{2}$. Frente a ello se ha observado en distintas unidades de cuidados intensivos (UCl) un distanciamiento de la enfermera intensivista con la familia, a diario el arduo quehacer, los múltiples cuidados que presenta el paciente crítico, el stress y otros dificultades que atraviesa la especialidad obstaculiza este vínculo, pero ello no debe justificar el quiebre de la relación enfermera - familia ya que enfermería como

\footnotetext{
Instituto Nacional Materno Perinatal

a Licenciada en Enfermería. Especialista en Cuidados Intensivos. Instituto Nacional Materno Perinatal.

Citar como: Zamora M, Meza O, Juárez E, Salazar M. Efecto de un programa educativo en el conocimiento y la incertidumbre de los familiares de pacientes en Unidad de Cuidados Intensivos Materna. Rev Peru Investig Matern Perinat 2019; 8(3):21-6

DOI https://doi.org/10.33421/inmp.2019161
}

Recibido: 26-09-2019 Aceptado: 30-09-2019 
profesión debe propiciar el cuidado integral a la familia, como esencia y razón de ser de la profesión ${ }^{3}$.

El ingreso a estas unidades generalmente se da en forma rápida y deja a la familia en la incertidumbre, esto produce ruptura de roles por lo que es esencial, que tanto el paciente como la familia reciban ayuda efectiva, al encontrarse en un ambiente altamente tecnificado y con procedimientos que parecen cruentos y amenazantes para ellos ${ }^{3}$.

La hospitalización de un familiar, altera el normal funcionamiento del núcleo familiar al que pertenece, se ha mostrado que los familiares experimentan niveles muy elevados de ansiedad y depresión mientras los pacientes están en la $\mathrm{UCl}^{1}$.

Si no se brinda el soporte oportuno al familiar, pueden generar estados ansiosos persistentes o conductas negativas hacia la UCl; por lo cual, es necesario educarlos para incorporarlos a este entorno que para ellos es hostil y atemorizante, ello debe ir precedido de información y educación, para disminuir la incertidumbre en el familiar ${ }^{4}$. El familiar del paciente crítico debe ser considerado como parte fundamental en el tratamiento y recuperación del paciente, ya que ellos pueden contribuir mejorando los resultados de su familiar hospitalizado 4 .

El objetivo de este proyecto de investigación se centra en evaluar el efecto de un programa educativo, en el nivel de conocimientos e incertidumbre de familiares de pacientes críticas de la Unidad de Cuidados Intensivos del Instituto Nacional Materno Perinatal, de esta forma darle un sentido humanizado a la labor que el enfermero de UCIM desarrolla.

\section{MATERIALES Y MÉTODOS}

Tipo de estudio: El presente estudio es de enfoque cuantitativo, diseño cuasi experimental por que evaluó el efecto de un programa educativo no aleatorizado con medición antes y después. Población de estudio En este estudio se tomó a toda la población mediante un muestreo no probabilístico por conveniencia, que estuvo conformada por familiares de las pacientes ingresadas a la UCI Materna, sea esposo, padre o madre de la paciente, identificado como cuidador principal el cual se definirá como la persona mayor de edad que, con lazos sanguíneos o no, se identifica como representante legal de ésta. Según las estadísticas de promedio de ingresos de pacientes en la unidad, la muestra estuvo conformada por 60 familiares que reunieron los criterios de inclusión y los cuales representan la totalidad de población en estudio. De ésta manera se utilizaron como criterio de inclusión los siguientes: Familiar del paciente crítico hospitalizado en la UCIM del INMP (papá, mamá, esposo) Identificado como cuidador principal de la paciente o identificado como representante legal de ésta, familiar cuidador principal cuyo paciente este sometido o no, a ventilación mecánica o con monitoreo invasivo, si la pareja es menor de edad debe haber firmado el asentimiento informado, familiar cuidador principal que desee participar libremente del estudio y ser la primera visita a su familiar. Recolección de datos. Antes de que los familiares ingresen a su primera visita a la $\mathrm{UCl}$, se le realizó un pre test para evaluar el grado de incertidumbre y el nivel de conocimientos que presentan en ese momento, luego se le orientó con el programa educativo en sus 4 fases, y al finalizar éste, se les realizó el post test. Para este estudio se empleó como técnica la encuesta; se recolectó los datos usando procedimientos estandarizados de manera que a cada participante se le haga las mismas preguntas de la misma manera. El instrumento fue un cuestionario previamente diseñado, dividido en 2 partes, la variable, incertidumbre fue medida a través de respuestas a los parámetros planteados en la Escala de Merle Mishel, modificado por los investigadores de acuerdo a la realidad de nuestra población y adaptado al familiar del paciente crítico, con alternativas de respuesta en las que el familiar respondió de acuerdo a como se sintió el momento de la evaluación. Cada pregunta tiene un puntaje en una escala que va desde, de acuerdo hasta no estoy de acuerdo, con un valor mínimo de 1 punto a un valor máximo de 3 puntos, a mayor puntaje mayor nivel de incertidumbre, El puntaje máximo de la escala es de 27 puntos y el mínimo 9 puntos. Y la variable conocimiento consta de 5 preguntas valoradas en 4 puntos cada respuesta correcta. Utilizado tanto para el pre test y el post test, con una duración máxima de 20 minutos. El instrumento fue sometido a evaluación por juicio de expertos, aprobando la validez y confiabilidad del instrumento, presentando un Alfa de Cronbach de $78.8 \%$.

\section{El puntaje para cada respuesta según la escala de incertidumbre de Merle Mishel}

\begin{tabular}{|l|l|}
\hline De acuerdo & 3 \\
\hline Indiferente & 2 \\
\hline No estoy de acuerdo & 1 \\
\hline
\end{tabular}

Puntaje mínimo de la escala 9 puntos

Puntaje máximo de la escala 27 puntos

Puntaje de corte de calificación:

- Puntaje de corte calificación Alto $\mathrm{NI}<10$ puntos

- Puntaje de corte calificación Regular $\mathrm{NI}=10-18$ puntos

- Puntaje de corte calificación Bajo NI >18 puntos

Para la variable, conocimiento que es la primera parte del cuestionario consta de 5 preguntas con 4 alternativas de respuesta cada una, donde solo una es la respuesta verdadera, las preguntas estarán referidas a los tópicos 
abordados en el programa educativo. Cada pregunta correcta tendrá el valor de 4 puntos.

Para clasificar las respuestas se utilizará la escala de Estaninos, que se muestra a continuación.

\begin{tabular}{|c|c|}
\hline Escala & Clasificación \\
\hline$<a$ & Bajo \\
\hline$a-b$ & Medio \\
\hline$>b$ & Alto \\
\hline
\end{tabular}

Se determinará los puntos de corte:

$A=$ promedio -0.75 * desv. Std

$\mathrm{B}=$ promedio $+0.75^{*}$ desv. Std

Análisis de datos. El análisis de los datos, estará compuesta por tres fases, la primera consistirá en identificar el nivel de clasificación para el conocimiento e incertidumbre de los familiares en el pre test generando los resúmenes descriptivos de frecuencias e indicadores estadísticos, luego se iniciará identificando el nivel de clasificación de las variables en las unidades de análisis, luego de ser sometidos al programa educativo; finalmente la tercera fase consistirá en la aplicación de una prueba de hipótesis el cual contrastará la presencia o no de diferencias significativas en los niveles de clasificación antes y después, y para tal efecto, se establecerá un nivel de confianza del 95\%, y como estadístico de prueba se utilizará la prueba de comparación de rangos de Wilcoxon, siendo esta un prueba no paramétrica.

\section{RESULTADOS}

En el estudio un total de 60 familiares de pacientes ingresados en la UCIM que reunieron los criterios de inclusión formaron parte del estudio y los cuales representan la totalidad de población, no rehusando ningún familiar a la realización de la misma. En cuanto a las características sociodemográficas de los familiares entrevistados, en la distribución por edades se encuentra que el mayor porcentaje $48 \%$ (29), está en el rango de edad de 25 a 54 años, que comprende la edad laboral máxima, y en menor porcentaje $7 \%$ (4) familiares se encuentran en la de edad de 65 años a más, que comprende el grupo de personas de edad avanzada. Según el sexo de los familiares coincidentemente hubo igual número de familiares entre sexo masculino y femenino. Los familiares encuestados en su mayoría 50\% (30) tenían como grado de instrucción el nivel secundario, pero un 7\% (4) no había culminado este nivel, un porcentaje alto también es el de nivel primario con $18 \%(11)$, mientras que el nivel técnico y superior suman un total de $24 \%$ (14). La mayoría de familiares 33\% (20) provienen del distrito de San Juan De Lurigancho, dentro de ellos 5 familiares eran de nacionalidad venezolana, el $15 \%$ (9) de familiares provenían de provincias entre los que destacan departamentos de Huánuco, cerro de Pasco, Ucayali, Chimbote, otros distritos de procedencia se calculan en un $30 \%$ (18). En cuanto al parentesco la mayoría $22 \%$ (13) era el esposo de la paciente, así como la madre $20 \%$ (12) en menor porcentaje cuñados de la paciente con $7 \%$ (4). De acuerdo al estado civil de los familiares de la paciente critica el $35 \%$ (21) son convivientes y un $34 \%$ (20) eran casados.

Al identificar el nivel de conocimientos en familiares de pacientes críticas antes del programa educativo tenemos que el mayor porcentaje $38.3 \%$ (23) familiares presentan un nivel de conocimiento medio, seguido de un $35 \%$ (21) familiares que presentan nivel de conocimiento alto, mientras que un $26.7 \%$ (16), presentan nivel de conocimiento bajo. Al identificar el nivel de incertidumbre en familiares de pacientes críticas antes del programa educativo tenemos un alto porcentaje $66.7 \%$ (40) de familiares que presentaron un alto nivel de incertidumbre, mientras que el $21.7 \%$ (13) familiares presentaron nivel de incertidumbre medio y tan solo un menor porcentaje $11.7 \%$ (7) presentaron nivel de incertidumbre bajo.

Al identificar el nivel de conocimientos en familiares de pacientes críticas después del programa educativo tenemos que el mayor porcentaje $73.3 \%$ (44) presenta nivel de conocimiento alto, seguido de un $21.7 \%$ (13) que presenta nivel de conocimiento medio, mientras que un menor porcentaje 5\% (3), presentó nivel de conocimiento bajo.

Al identificar el nivel de incertidumbre en familiares de pacientes críticas después del programa educativo tenemos que el mayor porcentaje $66.7 \%$ (40) presenta nivel de incertidumbre bajo, seguido de un $30 \%$ (18) que presenta nivel de incertidumbre medio, y un mínimo porcentaje $3.3 \%$ (2), presentó nivel de incertidumbre alto.

Al identificar el efecto del programa educativo en el nivel de conocimientos, tenemos 23 personas más con nivel de conocimiento alto, respecto al pre test. Lo que representa un incremento del 109.5\%. Así mismo tenemos 13 personas menos con nivel de conocimiento bajo, respecto al pre test. Lo que representa una disminución del $81.3 \%$.

Al identificar el efecto del programa educativo en el nivel de incertidumbre, Tenemos 38 personas menos con nivel de incertidumbre Alto, respecto al pre test. Lo que representa una disminución del 95\%. Así mismo tenemos 33 personas más con nivel de incertidumbre bajo, respecto al pre test. Lo que representa un incremento del $471.4 \%$.

Tabla 1. Efecto del programa educativo en el nivel de conocimiento en familiares de pacientes críticos.

\begin{tabular}{|c|c|c|c|c|c|c|}
\hline \multirow{2}{*}{$\begin{array}{c}\text { Nivel de } \\
\text { conocimiento }\end{array}$} & \multicolumn{2}{|c|}{ Pre test } & \multicolumn{2}{|c|}{ Post test } & \multirow{2}{*}{$\begin{array}{l}\text { Cant. } \\
\text { Perso }\end{array}$} & \multirow{2}{*}{$\begin{array}{c}\% \\
\text { Variación }\end{array}$} \\
\hline & $\mathbf{N}$ & $\%$ & $\mathbf{N}$ & $\%$ & & \\
\hline Conocimiento alto & 21 & $35.0 \%$ & 44 & $73.3 \%$ & 23 & $109.5 \%$ \\
\hline $\begin{array}{l}\text { Conocimiento } \\
\text { medio }\end{array}$ & 23 & $38.3 \%$ & 13 & $21.7 \%$ & -10 & $-43.5 \%$ \\
\hline Conocimiento bajo & 16 & $26.7 \%$ & 3 & $5.0 \%$ & -13 & $-81.3 \%$ \\
\hline Total & 60 & $100.0 \%$ & 60 & $100.0 \%$ & & \\
\hline
\end{tabular}


Tabla 2. Efecto del programa educativo en el nivel de incertidumbre en familiares de pacientes críticos.

\begin{tabular}{|c|c|c|c|c|c|c|}
\hline \multirow{2}{*}{$\begin{array}{c}\text { Nivel de } \\
\text { incertidumbre }\end{array}$} & \multicolumn{2}{|c|}{ Pre test } & \multicolumn{2}{|c|}{ Post test } & \multirow{2}{*}{$\begin{array}{l}\text { Cant. } \\
\text { Perso }\end{array}$} & \multirow{2}{*}{$\begin{array}{c}\% \\
\text { Variación }\end{array}$} \\
\hline & $\mathbf{N}$ & $\%$ & $\mathbf{N}$ & $\%$ & & \\
\hline Alto & 40 & $66.7 \%$ & 2 & $3.3 \%$ & -38 & $-95.0 \%$ \\
\hline Medio & 13 & $21.7 \%$ & 18 & $30.0 \%$ & 5 & $38.5 \%$ \\
\hline Bajo & 7 & $11.7 \%$ & 40 & $66.7 \%$ & 33 & $471.4 \%$ \\
\hline Total & 60 & $100.0 \%$ & 60 & $100.0 \%$ & & \\
\hline
\end{tabular}

En la figura 1, se puede observar la comparación de las distribuciones de puntuaciones de conocimiento obtenidas de las 60 personas participantes; en el pre test se observa que los resultados obtenidos fueron muy dispersos (desviación=5.38), es decir, que las notas obtenidas por los participantes estuvieron muy diferenciadas; algunos con notas aprobatorias llegando a puntaje máximo de 20 , mientras que otras con bajas calificaciones incluso llegando a obtener 0 puntos. Luego de la aplicación del programa, la distribución de las puntuaciones en el Post Test aumentó, mientras que su dispersión de puntuaciones disminuyó (desviación=3.18). Para comparar de forma puntual la efectividad de la aplicación del programa, solo basta en comparar las medianas de cada grupo (las rayas horizontales en la figura), siendo en el pre test igual a 12, mientras que en el post test llegó a 16.

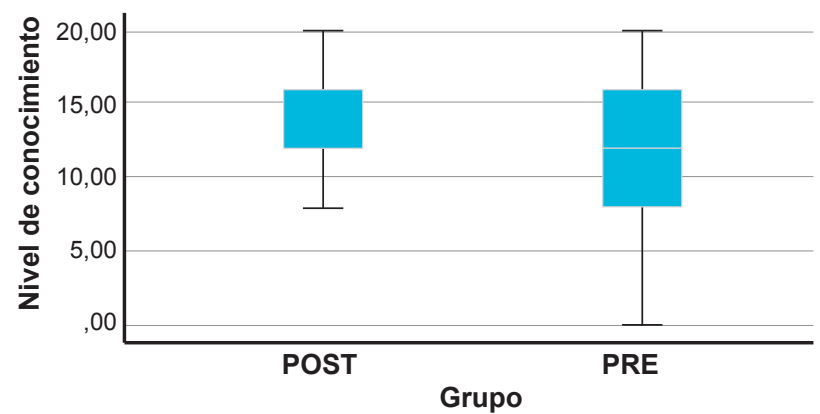

Figura 1. Comparación de puntuaciones en pre y post test, en el nivel de conocimiento

Tabla 3. Estadísticos descriptivos de las puntuaciones del nivel de conocimiento en el pre y post test.

\begin{tabular}{llc}
\hline Grupo & Medidas & Estadístico \\
\hline \multirow{4}{*}{ Pre test } & Media & 11.67 \\
& Mediana & 12.00 \\
& Desviación & 5.38 \\
& Mínimo & 0.00 \\
& Máximo & 20.00 \\
& Media & 15.60 \\
& Mediana & 16.00 \\
& Desviación & 3.18 \\
& Mínimo & 8.00 \\
& Máximo & 20.00 \\
\hline
\end{tabular}

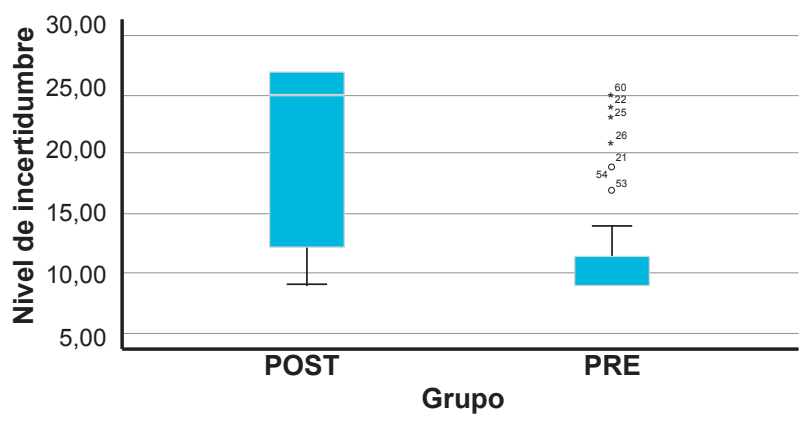

Figura 2. Comparación de puntuaciones en pre y post test, en el nivel de incertidumbre

En cuanto a la distribución de las puntuaciones sobre el nivel de incertidumbre, se identificó que en el pre test, fue poco disperso (desviación=4.18), mientras que el valor mediano de las puntuaciones de los participantes fue de 9 , además, sólo algunos de ellos obtuvieron notas superiores a 15. Luego, los resultados obtenidos en el post test, se obtuvo una mayor variación en las puntuaciones (desviación=6.80), algunos de los participantes mejoraron sus notas y otros las mantuvieron; comparando los resultados entre el pre y post test, la nota mediana en el post test fue de 25 , aumentando considerablemente en comparación con lo obtenido en el pre test.

En la tabla 5, para la comparación estadística fue necesario utilizar la prueba no paramétrica de muestras relacionadas, mediante la comparación de rangos de Wilcoxon, en el cual se estableció un nivel de significancia del $5 \%$. Para

Tabla 4. Estadísticos descriptivos de las puntuaciones del nivel de incertidumbre en el pre y post test.

\begin{tabular}{llc}
\hline Grupo & Medidas & Estadístico \\
\hline \multirow{4}{*}{ Pre test } & Media & 11.18 \\
& Mediana & 9.00 \\
& Desviación & 4.18 \\
& Mínimo & 9.00 \\
& Máximo & 25.00 \\
& Media & 21.22 \\
& Mediana & 25.00 \\
& Desviación & 6.80 \\
& Mínimo & 9.00 \\
& Máximo & 27.00 \\
\hline
\end{tabular}

Tabla 5. Prueba inferencial de comparación de medianas para el nivel de conocimiento e incertidumbre, en los grupos de Pre Test y Post Test.

\begin{tabular}{lcc}
\hline & $\begin{array}{c}\text { Nivel de conocimiento Pre } \\
\text { y Post }\end{array}$ & $\begin{array}{c}\text { Nivel de incertidumbre } \\
\text { Pre y Post }\end{array}$ \\
\hline$Z$ & $-4,341^{\mathrm{b}}$ & $-6,197^{\mathrm{b}}$ \\
Sig & 0.000 & 0.000 \\
\hline
\end{tabular}


el nivel de conocimiento, se obtuvo un valor de $Z$ igual a -4.34 , y un sig menor al $5 \%$, con lo que se establece que hay diferencias significativas entre las medianas obtenidas en el pre y post test, concluyéndose que el programa aplicado fue efectivo para mejorar el conocimiento de los participantes. El análisis para la comparación entre el pre y post, en el nivel de incertidumbre, se obtuvo un valor de $Z$ igual a -6.20 y un sig menor al $5 \%$, evidenciándose una diferencia estadísticamente significativa entre los valores medianos del pre y post test, con el cual se llega a la conclusión de que el programa aplicado fue efectivo para mejorar el nivel de in certidumbre de los participantes.

\section{DISCUSIÓN}

Los resultados del estudio afirman la importancia de la aplicación de un programa educativo dirigido a los familiares de pacientes ingresados en una unidad de cuidados intensivos, en efecto la información diaria brindada en dicho programa es muy bien valorada por el familiar, la comunicación con los familiares de las pacientes críticas debería ser evaluada con regularidad con la intención de mejorarla, ya que es muy importante para que en un momento dado se puedan tomar decisiones en conjunto en relación con el tratamiento a llevar a cabo; todo ello evitaría en cierta medida los síntomas de estrés postraumático que desarrollan muchos de los familiares de los pacientes críticos 5 .

Debemos tener en cuenta que la comunicación marca la diferencia durante el proceso de información y así lo evidencian estudios previos, pero para que ésta sea eficaz, debe transmitir un mensaje que pueda ser entendido y recordado ${ }^{6,7}$. Muchos de los errores en el proceso de información son causados por faltas de comprensión y memoria, por ello la necesidad de incluir una maqueta didáctica en este programa, lo cual hizo más comprensible y fácilmente interpretable los conocimientos brindados ${ }^{7}$

El equipo multidisciplinario que atiende al paciente $o$ a su familiar debería aprender a escuchar para ser capaz de responder a las señales no verbales, y no utilizar terminología médica, ya que esto facilitaría con más rapidez el contacto con el paciente y sus familiares.

La comunicación y las relaciones que se establecen entre el equipo de salud, los pacientes y su familia tienen que cambiar si queremos humanizar los cuidados en las unidades de cuidados intensivos. Queda visible la importancia de la información y educación en el familiar de la paciente crítica, pudiendo ser definida como una necesidad básica de la familia, tal como otros autores ya lo han descrito ${ }^{1,8,6}$.

La educación en el familiar es tan importante que existen estudios que han desarrollado interesantes programas de información y educación para los familiares de pacientes ingresados en $\mathrm{UCI}^{1,4,9,10}$.
En la presente investigación se evidenció que crear un programa educativo protocolizando la acogida del familiar en la UCIM fue muy positiva, ya que después del programa educativo el nivel de conocimientos es alto y el nivel de incertidumbre es bajo en los familiares de los pacientes ingresados a UCIM. Por otro lado, deducimos que la información que se proporciona a través de éste programa responde a las necesidades de los familiares y se sienten gratificados con el grado de información.

Antes de la realización del programa educativo el $88 \%$ de los familiares no habían sido informados del diagnóstico de su familiar antes del ingreso a la UCIM; esto puede deberse a que la mayoría de pacientes ingresa por emergencia a sala de operaciones y pocas veces el cirujano tiene contacto directo con el esposo o familiar directo para brindarle información del estado de salud de su familiar. Esto debería ser tomado en cuenta pues como ya estudios lo evidencian la intensidad de la experiencia que presenta la familia, cuando una paciente es ingresada a la unidad de cuidados intensivos implica estrés tanto para la paciente que muchas veces deja a los hijos pequeños en el hogar, como a sus familiares que ve interrumpido sus roles cotidianos, estrés que está originado no sólo por su gravedad, sino también por las dificultades en la comunicación que existen con la paciente y su familia ${ }^{11}$.

En conclusión, el programa educativo mejora significativamente el nivel de conocimientos y disminuye el nivel de incertidumbre en familiares de pacientes críticas de la unidad de cuidados intensivos. Éstos resultados son de gran importancia para el profesional que labora en la unidad de cuidados intensivos, ya que permite implementar intervenciones dirigidas a mejorar el nivel de conocimientos y así mismo disminuir el nivel de incertidumbre, el hecho de saber ¿Qué es la UCl?, el personal de salud que atiende a su familiar, el uso del equipo de monitoreo y los múltiples invasivos colocados en su paciente y el hecho de que sepan que las necesidades de su familiar están siendo satisfechas, permite establecer medidas efectivas para su disminución, del mismo modo un buen informe del diagnóstico, tratamiento, medidas de soporte vital y evolución de la enfermedad explicada por el galeno en términos de fácil comprensión contribuyen al proceso de humanización de los cuidados intensivos . Por este motivo se recomienda implementar el programa educativo con información clara, de calidad y que cubra todos los aspectos que quiere conocer el entorno de los pacientes.

Financiamiento: Autofinanciado.

Conflicto de intereses: Los autores declaran no presentar conflicto de intereses en la presente investigación.

\section{REFERENCIAS BIBLIOGRÁFICAS}

1. Torrents R, Oliva E, Torras M, Saucedo F, Suroca L, et al. Impacto de los familiares del paciente critico ante una acogida 
protocolizada [tesis]. Universidad Autónoma de Barcelona, facultad de enfermería; 2004.

2. Marrero M. La familia como objeto de estudio en el proceso de salud-enfermedad de los trabajadores. Rev Cubana salud trabajo. 2004; 5(1)

3. Charry R, Ordoñez L. Tiempos M. Interacción entre el profesional de enfermería y la familia del paciente hospitalizado en la unidad de cuidados intensivos. [tesis]. Bogotá: Universidad de La Sabana, Facultad de Enfermería; 2012

4. Giraldo N, García J. Cuidado para el bienestar de los familiares de pacientes hospitalizados en una unidad de cuidado intensivo adultos con base en la teoría de Kristen Swanson. [Tesis]. Bogotá: Universidad Nacional De Colombia. Facultad De Enfermería Cardiorrespiratoria; 2010.

5. Pardavila B, Vivar C. Necesidades de la familia en las unidades de cuidados intensivos. Revisión de la literatura. Enferm Intensiva. 2012;23(2):51-67

6. Llamas F, Flores J, Acosta M, González J, et al. Necesidades de los familiares en una Unidad de Cuidados Críticos. Rev Enferm intensiva. 2012; 23(2): 51-67

7. Pérez T, Ferreiro I, Pigem R. Las maquetas como material didáctico para la enseñanza y aprendizaje [tesis]. España: Universidad de Alicante, Departamento de expresión gráfica y cartografía; 2013
8. Uribe MT, Muñoz C. Restrepo J. Percepción familiar del paciente crítico cardiovascular. [tesis]. Antioquia: Universidad de Antioquia, Facultad de Enfermería ; 2004

9. Garcia J, et al. Efectividad del modelo de cuidado de enfermería para la familia de paciente con infarto agudo al miocardio en la unidad de cuidados intensivos. Rev Cuid. 2015; 6(1): 923-931.

10. Becerra F, Castro E, Suarez L, Espitia Z. Programa de educación a familiares de pacientes en unidades de cuidados intensivos adultos: “UCl amiga acompáñame". [Tesis].Bogotá: Universidad de la Sabana, Facultad de Enfermería; 2011

11. Brevis Y. Creencias, sentimientos e incertidumbre frente a la enfermedad en familiares de Pacientes ingresados a $\mathrm{UCl}$. Rev Chil Medicina Inten. 2011; 26(1): 27-34

Correspondencia: Mariela Zamora Córdova.

Dirección: Instituto Nacional Materno Perinatal. Jirón Miro Quesada 941Cercado de Lima 15001 Perú.

Correo: mariela.zamora.c@upch.pe 